\title{
ON SOME TURÁN-TYPE INEQUALITIES
}

\author{
A. LAFORGIA AND P. NATALINI
}

Received 14 September 2005; Accepted 20 September 2005

We prove Turán-type inequalities for some special functions by using a generalization of the Schwarz inequality.

Copyright (C 2006 A. Laforgia and P. Natalini. This is an open access article distributed under the Creative Commons Attribution License, which permits unrestricted use, distribution, and reproduction in any medium, provided the original work is properly cited.

\section{Introduction}

The importance, in many fields of mathematics, of the inequalities of the type

$$
f_{n}(x) f_{n+2}(x)-f_{n+1}^{2}(x) \leq 0,
$$

where $n=0,1,2, \ldots$, is well known. They are named, by Karlin and Szegö, Turán-type inequalities because the first of this type of inequalities was proved by Turán [12]. More precisely, by using the classical recurrence relation [10, page 81 ]

$$
\begin{gathered}
(n+1) P_{n+1}(x)=(2 n+1) x P_{n}(x)-n P_{n-1}(x), \quad n=0,1, \ldots \\
P_{-1}(x)=0, \quad P_{0}(x)=1
\end{gathered}
$$

and the differential relation [10, page 83$]$

$$
\left(1-x^{2}\right) P_{n}^{\prime}(x)=n P_{n-1}(x)-n x P_{n}(x),
$$

he proved the following inequality:

$$
\left|\begin{array}{cc}
P_{n}(x) & P_{n+1}(x) \\
P_{n+1}(x) & P_{n+2}(x)
\end{array}\right| \leq 0, \quad-1 \leq x \leq 1,
$$

where $P_{n}(x)$ is the Legendre polynomial of degree $n$. In (1.4) equality occurs only if $x= \pm 1$. This classical result has been extended in several directions: ultraspherical polynomials, Laguerre and Hermite polynomials, Bessel functions of first kind, modified Bessel functions, and so forth. 
For example, Lorch [8] established Turán-type inequalities for the positive zeros $c_{v k}$, $k=1,2, \ldots$, of the general Bessel function

$$
C_{\nu}(x)=J_{\nu}(x) \cos \alpha-Y_{\nu}(x) \sin \alpha, \quad 0 \leq \alpha<\pi,
$$

where $J_{v}(x)$ and $Y_{v}(x)$ denote the Bessel functions of the first and the second kind, respectively, while the corresponding results for the positive zeros $c_{v k}^{\prime}, v \geq 0, k=1,2, \ldots$, of the derivative $C_{\nu}^{\prime}(x)=(d / d x) C_{\nu}(x)$ and for the zeros of ultraspherical, Laguerre, and Hermite polynomials have been established in $[2,3,6]$, respectively.

Recently, in [7], we have proved Turán-type inequalities for some special functions, as well as the polygamma and the Riemann zeta functions, by using the following generalization of the Schwarz inequality:

$$
\int_{a}^{b} g(t)[f(t)]^{m} d t \cdot \int_{a}^{b} g(t)[f(t)]^{n} d t \geq\left[\int_{a}^{b} g(t)[f(t)]^{(m+n) / 2} d t\right]^{2},
$$

where $f$ and $g$ are two nonnegative functions of a real variable and $m$ and $n$ belong to a set $S$ of real numbers, such that the integrals in (1.6) exist.

As mentioned in [7] this approach represents an alternative method with respect to the classical ones used by the above-cited authors and based, prevalently, on the Sturm theory.

In this paper, we continue, in this direction, to investigate about Turán-type inequalities satisfied by some special functions. In the next section, we will give three results. In the first one, we will use the well-known psi function defined as follows:

$$
\psi(x)=\frac{\Gamma^{\prime}(x)}{\Gamma(x)}, \quad x>0
$$

with the usual notation for the gamma function.

In the second one, we will use the so-called Riemann $\xi$-function which can be defined (see [11, page 16], cf. [9, page 285]) by

$$
\xi(s)=\frac{1}{2} s(s-1) \pi^{-s / 2} \Gamma\left(\frac{s}{2}\right) \zeta(s),
$$

where $\zeta$ is the Riemann $\zeta$-function. This function has the following representation (see [5]):

$$
\xi\left(s+\frac{1}{2}\right)=\sum_{k=0}^{\infty} b_{k} s^{2 k}
$$

where the coefficients $b_{k}$ are given by the formula

$$
\begin{aligned}
& b_{k}=8 \frac{2^{2 k}}{(2 k) !} \int_{0}^{\infty} t^{2 k} \Phi(t) d t, \quad k=0,1, \ldots, \\
& \Phi(t)=\sum_{n=1}^{\infty}\left(2 \pi^{2} n^{4} e^{9 t}-3 \pi n^{2} e^{5 t}\right) e^{-\pi n^{2} e^{4 t}} .
\end{aligned}
$$


In [1] the following Turán-type inequalities were proved:

$$
b_{k}^{2}-\frac{k+1}{k} b_{k+1} b_{k-1} \geq 0, \quad k=0,1, \ldots,
$$

which are very important in the theory of the Riemann $\xi$-function (see [5]).

In the third one, we will use the modified Bessel functions of the third kind $K_{v}(x)$, $x>0$, defined as follows:

$$
\begin{gathered}
K_{v}(x)=\frac{\pi}{2} \frac{I_{-\nu}(x)-I_{\nu}(x)}{\sin \nu \pi}, \quad v \neq 0, \pm 1, \pm 2, \ldots, \\
K_{n}(x)=\lim _{\nu \rightarrow n} K_{\nu}(x), \quad n=0, \pm 1, \pm 2, \ldots,
\end{gathered}
$$

where

$$
I_{\nu}(x)=\sum_{k=0}^{\infty} \frac{(x / 2)^{\nu+2 k}}{k ! \Gamma(\nu+k+1)}
$$

are the modified Bessel functions of the first kind.

\section{The results}

Theorem 2.1. For $n=1,2, \ldots$, denote by $h_{n}=\sum_{k=1}^{n}(1 / k)$ the partial sum of the harmonic series. Let

$$
a_{n}=h_{n}-\log n
$$

then

$$
\left(a_{n}-\gamma\right)\left(a_{n+2}-\gamma\right) \geq\left(a_{n+1}-\gamma\right)^{2},
$$

where $\gamma$ is the Euler-Mascheroni constant defined by

$$
\gamma=-\psi(1)=0,5772156649 \ldots
$$

Proof. For the psi function, we use the following expression:

$$
\psi(n+1)=\sum_{k=1}^{n} \frac{1}{k}-\gamma, \quad n=1,2, \ldots,
$$

and the following integral representation:

$$
\psi(z+1)=\int_{0}^{\infty}\left(\frac{e^{-t}}{t}-\frac{e^{-z t}}{e^{-t}-1}\right) d t, \quad \operatorname{Re} z>0 .
$$

By putting $z=n$ in (2.5), for $n=1,2, \ldots$, we obtain from (2.4) and (2.5),

$$
\sum_{k=1}^{n} \frac{1}{k}-\gamma=\int_{0}^{\infty}\left(\frac{e^{-t}}{t}-\frac{e^{-n t}}{e^{-t}-1}\right) d t=\int_{0}^{\infty} \frac{e^{-t}-e^{-n t}}{t} d t+\int_{0}^{\infty} e^{-n t} \frac{e^{t}-1-t}{t\left(e^{t}-1\right)} d t
$$


4 On some Turán-type inequalities

Since

$$
\int_{0}^{\infty} \frac{e^{-t}-e^{-n t}}{t} d t=\log n
$$

we have

$$
\sum_{k=1}^{n} \frac{1}{k}-\log n-\gamma=\int_{0}^{\infty} \frac{e^{t}-1-t}{t\left(e^{t}-1\right)} e^{-n t} d t
$$

By (1.6) with $g(t)=\left(e^{t}-1-t\right) / t\left(e^{t}-1\right), f(t)=e^{-t}$ and $a=0, b=+\infty$, we get

$$
\int_{0}^{\infty} \frac{e^{t}-1-t}{t\left(e^{t}-1\right)} e^{-n t} d t \cdot \int_{0}^{\infty} \frac{e^{t}-1-t}{t\left(e^{t}-1\right)} e^{-(n+2) t} d t \geq\left[\int_{0}^{\infty} \frac{e^{t}-1-t}{t\left(e^{t}-1\right)} e^{-(n+1) t} d t\right]^{2}
$$

that is the inequality (2.2).

Theorem 2.2. For $k=1,2, \ldots$, let $b_{k}(k=1,2, \ldots)$ be the coefficients in (1.9), then

$$
b_{k}^{2}-\frac{(2 k+1)(k+1)}{k(2 k-1)} b_{k+1} b_{k-1} \leq 0, \quad k=1,2, \ldots
$$

Proof. By (1.6) and (1.10), with $g(t)=8 \Phi(t), f(t)=(2 t)^{2}$ and $a=0, b=+\infty$, we get

$$
\int_{0}^{\infty} 8 \Phi(t)(2 t)^{2 k+2} d t \cdot \int_{0}^{\infty} 8 \Phi(t)(2 t)^{2 k-2} d t \geq\left[\int_{0}^{\infty} 8 \Phi(t)(2 t)^{2 k} d t\right]^{2} .
$$

Dividing (2.11) by $(2 k)$ ! this inequality becomes

$$
\frac{(2 k+2) !}{(2 k) !} b_{k+1} \frac{(2 k-2) !}{(2 k) !} b_{k-1} \leq b_{k}^{2}, \quad k=1,2, \ldots,
$$

from which, since $((2 k+2) ! /(2 k) !)((2 k-2) ! /(2 k) !)=((2 k+1)(k+1)) / k(2 k-1)$, we obtain the conclusion of Theorem 2.2.

Remark 2.3. It is important to note that inequalities (1.12) and (2.10) together give

$$
\frac{k+1}{k} b_{k+1} b_{k-1} \leq b_{k}^{2} \leq \frac{k+1}{k} \frac{2 k+1}{2 k-1} b_{k+1} b_{k-1}, \quad k=1,2, \ldots
$$

Theorem 2.4. Let $K_{v}(x), x>0$, be the modified Bessel function of the third kind. Then, for $\nu>-1 / 2$ and $\mu>-1 / 2$,

$$
K_{\nu}(x) \cdot K_{\mu}(x) \geq K_{(\nu+\mu) / 2}^{2}(x) .
$$

Proof. By (1.6) with $g(t)=e^{-\beta / t-\gamma t}, f(t)=t^{-1}$ and $a=0, b=+\infty$, we get

$$
\int_{0}^{\infty} t^{m-1} e^{-\beta / t-\gamma t} d t \cdot \int_{0}^{\infty} t^{n-1} e^{-\beta / t-\gamma t} d t \geq\left[\int_{0}^{\infty} t^{(m+n) / 2-1} e^{-\beta / t-\gamma t} d t\right]^{2} .
$$


Using the following formula (see [4, Integral 3.471(9)]):

$$
\int_{0}^{\infty} t^{\nu-1} e^{-\beta / t-\gamma t} d t=2\left(\frac{\beta}{\gamma}\right)^{\nu / 2} K_{\nu}(2 \sqrt{\beta \gamma}), \quad \nu>-\frac{1}{2}
$$

from (2.15) we have

$$
K_{\nu}(2 \sqrt{\beta \gamma}) \cdot K_{\mu}(2 \sqrt{\beta \gamma}) \geq K_{(\nu+\mu) / 2}^{2}(2 \sqrt{\beta \gamma})
$$

which, putting $x=2 \sqrt{\beta \gamma}$, is equivalent to the conclusion of Theorem 2.4.

In the particular case $\mu=\nu+2$, we find

$$
K_{\nu}(x) \cdot K_{\nu+2}(x) \geq K_{\nu+1}^{2}(x), \quad v>-\frac{1}{2} .
$$

Concluding Remark 2.5. By means of (1.6) Turán-type inequalities for many complicated integrals as well as, for example, $s_{n}=\int_{0}^{\pi}(\log \sin x)^{n} d x(n=0,1, \ldots)$ for which we have

$$
s_{n}(x) s_{n+2}(x) \geq s_{n+1}^{2}(x)
$$

can be obtained.

\section{References}

[1] G. Csordas, T. S. Norfolk, and R. S. Varga, The Riemann hypothesis and the Turán inequalities, Transactions of the American Mathematical Society 296 (1986), no. 2, 521-541.

[2] Á. Elbert and A. Laforgia, Some monotonicity properties of the zeros of ultraspherical polynomials, Acta Mathematica Hungarica 48 (1986), no. 1-2, 155-159.

[3] __ Monotonicity results on the zeros of generalized Laguerre polynomials, Journal of Approximation Theory 51 (1987), no. 2, 168-174.

[4] I. S. Gradshteyn and I. M. Ryzhik, Table of Integrals, Series, and Products, 6th ed., Academic Press, California, 2000.

[5] O. M. Katkova, Multiple positivity and the Riemann zeta-function, preprint, 2005, http://arxiv. org/abs/math.CV/0505174.

[6] A. Laforgia, Sturm theory for certain classes of Sturm-Liouville equations and Turánians and Wronskians for the zeros of derivative of Bessel functions, Indagationes Mathematicae 44 (1982), no. 3, 295-301.

[7] A. Laforgia and P. Natalini, Turán-type inequalities for some special functions, to appear in Journal of Inequalities in Pure and Applied Mathematics.

[8] L. Lorch, Turánians and Wronskians for the zeros of Bessel functions, SIAM Journal on Mathematical Analysis 11 (1980), no. 2, 223-227.

[9] G. Pólya, Collected Papers. Vol. II: Location of Zeros, edited by R. P. Boas, Mathematicians of Our Time, vol. 8, The MIT Press, Massachusetts, 1974.

[10] G. Szegö, Orthogonal Polynomials, 4th ed., Colloquium Publications, vol. 23, American Mathematical Society, Rhode Island, 1975. 
6 On some Turán-type inequalities

[11] E. C. Titchmarsh, The Theory of the Riemann Zeta-Function, The Clarendon Press, Oxford, 1951.

[12] P. Turán, On the zeros of the polynomials of Legendre, Časopis Pro Pěstování Matematiky 75 (1950), 113-122.

A. Laforgia: Department of Mathematics, Roma Tre University, Largo San Leonardo Murialdo, 100146 Rome, Italy

E-mail address: laforgia@mat.uniroma3.it

P. Natalini: Department of Mathematics, Roma Tre University, Largo San Leonardo Murialdo, 100146 Rome, Italy

E-mail address: natalini@mat.uniroma3.it 